\title{
Distinct DNA methylation alterations are associated with cribriform architecture and intraductal carcinoma in Gleason pattern 4 prostate tumors
}

\author{
EKATERINA OLKHOV-MITSEL ${ }^{1,2}$, FARSHID SIADAT $^{3}$, KEN KRON $^{4}$, LIYANG LIU $^{1,2}$, ANDREA J. SAVIO $^{1,2}$, \\ JOHN TRACHTENBERG ${ }^{5}$, NEIL FLESHNER ${ }^{5}$, THEODORUS VAN DER KWAST ${ }^{2,6}$ and BHARATI BAPAT ${ }^{1,2,6}$
}

${ }^{1}$ Lunenfeld-Tanenbaum Research Institute, Mount Sinai Hospital, Toronto, ON M5T3L9; ${ }^{2}$ Department of Laboratory Medicine and Pathobiology, University of Toronto, Toronto, ON M5S1A1; ${ }^{3}$ Department of Anatomical Pathology, Royal Alexandra Hospital, Edmonton, AB T5H3V9; ${ }^{4}$ Ontario Cancer Institute, Princess Margaret Cancer Center, University Health Network, Toronto, ON M5G0A3; ${ }^{5}$ Division of Urology, Department of Surgical Oncology, University Health Network, University of Toronto, Toronto, ON M5G2M9; ${ }^{6}$ Department of Pathology, University Health Network, Toronto, ON M5G2C4, Canada

Received September 23, 2016; Accepted February 23, 2017

DOI: $10.3892 / \mathrm{ol} .2017 .6140$

\begin{abstract}
The aim of the present study was to explore DNA methylation aberrations in association with cribriform architecture and intraductal carcinoma (IDC) of the prostate, as there is robust evidence that these morphological features are associated with aggressive disease and have significant clinical implications. Herein, the associations of a panel of seven known prognostic DNA methylation biomarkers with cribriform and IDC features were examined in a series of 91 Gleason pattern (GP) 4 tumors derived from Gleason score 7 radical prostatectomies. Gene specific DNA methylation was compared between cribriform and/or IDC positive vs. negative cases, and in association with clinicopathological features, using Chi square and Mann-Whitney U tests. DNA methylation of the adenomatous polyposis coli, Ras association domain family member 1 and T-box 15 genes was significantly elevated in GP4 tumors with cribriform and/or IDC features compared
\end{abstract}

Correspondence to: Dr Bharati Bapat, Lunenfeld-Tanenbaum Research Institute, Mount Sinai Hospital, 60 Murray Street, Toronto, ON M5T3L9, Canada

E-mail: bapat@lunenfeld.ca

Abbreviations: APC, adenomatous polyposis coli; CYP26A1, cytochrome P450 family 26 subfamily A member 1; FFPE, formalin-fixed paraffin-embedded; GP, Gleason pattern; GS, Gleason score; H\&E, hematoxylin and eosin; HM, high methylation; HOX, homeobox; IDC, intraductal carcinoma; LM, low methylation; $\mathrm{PCa}$, prostate cancer; PMR, percent methylation ratio; PSA, prostate-specific antigen; RASSF1, Ras association domain family member 1; RP, radical prostatectomy; TBX15, T-box 15; TGF, intraductal carcinoma; LM, $10 \beta 2$

Key words: prostate cancer, DNA methylation, biomarker, MethyLight, pathology, cribriform architecture, intraductal carcinoma, Gleason pattern with negative cases $(\mathrm{P}=0.045, \mathrm{P}=0.007$ and $\mathrm{P}=0.013$, respectively). To the best of our knowledge, this provides the first evidence for an association between cribriform and/or IDC and methylation biomarkers, and warrants further investigation of additional DNA methylation events in association with various architectural patterns in prostate cancer.

\section{Introduction}

Prostate cancer (PCa) is the second most common type of cancer affecting men worldwide, with an incidence of 1.1 million new cases each year (1). The most widely accepted pathological grading of $\mathrm{PCa}$ is the Gleason Score (GS), which is based on architectural features of the gland and is comprised of the sum of two Gleason Patterns (GP) (2). The GS grading system is among the most effective predictors of clinical outcomes following radical prostatectomy (RP), and is universally used to guide PCa treatment (3). Within this system, GS6 PCas are low grade, whereas GS8 or higher are classified as high grade. GS7 prostate adenocarcinomas consisting of variable proportions of GP3 and GP4 are considered to be intermediate grade, and represent the most heterogeneous group of neoplasms with diverse clinical outcomes $(4,5)$ Thus, GS7 PCa is the focus of the current study, as identifying specific prognostic factors that can provide additional information on disease progression and treatment outcomes for patients with GS7 PCa would be of marked clinical benefit.

GP4 is assigned to adenocarcinomas when they contain any of the following architectural patterns: Small/large fused glands, poorly formed glands, glomeruloid and cribriform architecture (4-6). The cribriform pattern is commonly associated with intraductal carcinoma (IDC), a distinct histopathological entity characterized by malignant cells spanning the lumen of prostate ducts and acini (7-9). Although specific morphologies of $\mathrm{PCa}$, including cribriform architecture and IDC, have been recognized for decades, their independent clinical significance is only now emerging (8). Studies have demonstrated that IDC in biopsy and/or RP tissues is indicative 
of a possible adverse clinical course and metastatic disease, warranting further aggressive treatment (10-13). Similarly, cribriform architecture has been demonstrated to be a clinically significant independent prognosticator for PCa-specific mortality (14-17).

The unequivocal identification of these pathological features based on the morphological criteria alone has been reported as challenging $(18,19)$. In this regard, molecular features such as DNA methylation alterations may be of value. Aberrations in DNA methylation deregulate the genome and contribute to the loss of tissue homeostasis observed in aging and in diseases such as PCa (20). They may constitute the driver and the passenger events of tumorigenesis, as is evident from the widespread hypermethylation at the promoter regions of tumor suppressor genes, which is a hallmark of PCa $(20,21)$. Detection of tumor-specific DNA methylation alterations in biopsy tissues may, in the future, serve as molecular indicators of cribriform architecture and/or of IDC. Additionally, DNA methylation markers are emerging as promising biomarkers of prostate carcinogenesis, thus complementing, if not altogether avoiding, the requirement for the histopathological examination of biopsy tissue samples (22). The results of our previous studies demonstrated that the DNA hypermethylation of a panel of seven genes [adenomatous polyposis coli $(A P C)$, cytochrome $\mathrm{P} 450$ family 26 subfamily A member 1 (CYP26A1), homeobox D3 (HOXD3), HOXD8, Ras association domain family member 1 (RASSF1), T-box 15 (TBX15) and transforming growth factor- $\beta 2$ $(T G F \beta 2)]$ is associated with PCa disease progression and clinical outcome (23-25). These markers were initially identified through a genome-wide methylation screen of low-grade and high-grade PCas (23). Subsequently, the prognostic potential of a subset of these markers was validated in an independent cohort $(24,25)$. However, to the best of our knowledge, their association with the tumor architectural features of aggressive PCa has not yet been investigated.

In the present study, cribriform architecture and IDC were characterized in GP4 tumors derived from GS7 RP specimens, and their association with the aforementioned panel of seven DNA methylation markers was examined.

\section{Materials and methods}

Clinical samples and information. The present study was approved by the University Health Network Research Ethics Board. Retrospective formalin-fixed paraffin-embedded (FFPE) RP specimens from a total of 91 patients diagnosed with PCa between 1998 and 2001 at the University Health Network were included in the current study. This is a subset of a larger cohort of 246 patients (consisting of 91 GS7 tumors) that was characterized in our previous study (17). As previously reported, GS $\geq 8$ tumors were limited in the present cohort and, thus, were excluded from analysis (17). The clinical and pathological characteristics of the cohort are listed in Table I. All samples, as well as clinical and pathological follow-up data, were obtained according to protocols approved by the Research Ethics Board of Mount Sinai Hospital, Toronto, and the University Health Network (Toronto, Canada).

Histological evaluation. The complete set of hematoxylin and eosin (H\&E)-stained slides from each RP tissue sample was

Table I. Clinicopathological characteristics of the study cohort.

Clinicopathological characteristics

Total number of patients

Gleason score ${ }^{\mathrm{a}}$, no. of patients

$7(3+4)$

$7(4+3)$

Pathological stage, no. of patients

pT2

pT3a

pT3b

pT4

Surgical margin status, no. of patients

Negative

Positive

Average preoperative PSA ${ }^{b}$

Average prostate weight, grams ${ }^{\mathrm{b}}$

Median age, years

aSUP 2005 GS assigned by an expert genitourinary pathologist; ${ }^{\mathrm{b}} \mathrm{n}=85$. PSA, prostate-specific antigen.

A

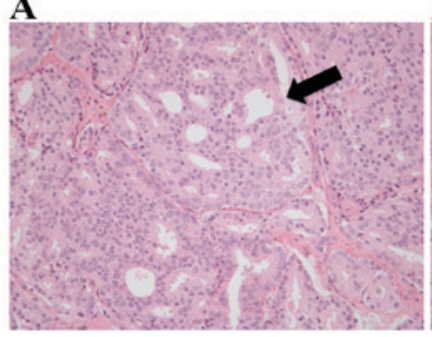

B

Figure 1. Representative hematoxylin and eosin stains of prostate cancer Gleason pattern 4 tissues with (A) cribriform architecture (marked with a black arrow) and (B) intraductal carcinoma (marked with a blue arrow). Magnification, $\mathrm{x} 100$.

collected and reviewed by an expert genitourinary pathologist in order to assign a modified GS (International Society of Urological Pathology 2005), along with the pathological stage, prostate weight and surgical margin status (4). For each GS7 $\mathrm{PCa}$, the most representative GP4 tumor regions were marked on the H\&E-stained slides corresponding to an area of $\geq 80 \%$ neoplastic cellularity, and the cells isolated from these regions were used for DNA methylation analysis. Only pure GP4 tissue was analyzed. The same marked areas on the H\&E-stained slides were also independently reviewed by another genitourinary pathologist for the presence or absence of cribriform architecture, which is characterized by confluent epithelial proliferation with multiple lumina without intervening stroma, and of IDC, which is defined as a lumen-spanning solid or cribriform expansile neoplastic proliferation within the prostate gland or ducts (Fig. 1). Therefore, the presence of cribriform architecture and IDC in the same GP4 area was investigated, an area that was manually macrodissected using a scalpel for subsequent DNA methylation analysis, as described previously (23-25). All 
Table II. Primer and probe sequences used in the MethyLight assay for APC, CYP26A1, HOXD3, HOXD8, RASSF1, TBX15, $T G F-\beta$ genes and $A L U$ control reaction.

$\mathrm{Gene}^{\mathrm{a}, \mathrm{b}} \quad$ Sequence

$A L U$

$A P C$

ENSG000001349821

Chr5: 112737781-1127378562

CYP26A1

ENSG00000095596

Chr10: 93069103-93069230

HOXD3

ENSG00000128652

Chr2: 176163186-176163274

HOXD8

ENSG00000175879

Chr2: 176128934-176129040

RASSF 1

ENSG00000068028

Chr3: 50340720-503407843

TBX15

ENSG00000092607

Chr1: 118984438-118984535

$T G F-\beta$

ENSG00000092969

Chr1: 218346933-218347007
Forward: 5'-GGTTAGGTATAGTGGTTTATATTTGTAATTTTAGTA-3'

Reverse: 5'-ATTAACTAAACTAATCTTAAACTCCTAACCTCA-3'

Probe: 5'FAM-CCTACCTTAACCTCCC-3'BHQ1

Forward: 5'-GAACCAAAACGCTCCCCAT-3'

Reverse: 5'-TTATATGTCGGTTACGTGCGTTTATAT-3'

Probe: 5'FAM-CCCGTCGAAAACCCGCCGATTA-3'BHQ1

Forward: 5'-TTGTAGAGATTCGACGTACGCGG-3'

Reverse: 5'-AAAACCTTCCGTCAAACATCCTCTACG-3'

Probe: 5'FAM-ACGCCCACGACGTACCCGCTTCCTTAC-3'BHQ1

Forward: 5'-TTAAAGGTTTATGGTTGCGC-3'

Reverse: 5'-TTACGAACACTAAACTACACCCG-3'

Probe: 5'FAM-ACAAAACGTTCCCGACGCTTCTAAAA-3'BHQ1

Forward: 5'-TAGTCGGTTTTGGTTCGTTGC-3'

Reverse: 5'-CGTTCTAAAACGAAAAAAAAAACTCGCG-3'

Probe: 5'FAM-TCCTCGAACAAAACGCGACTCCCGAATCTC-3'BHQ1

Forward: 5'-ATTGAGTTGCGGGAGTTGGT-3'

Reverse: 5'-ACACGCTCCAACCGAATACG-3'

Probe: 5'FAM-CCCTTCCCAACGCGCCCA-3'BHQ1 ${ }^{\mathrm{c}}$

Forward: 5'-GCGGTTTTGTAAGTATATTGTTGCG-3'

Reverse: 5'-ACTCCGAATAAAACAAAAACTAAAATCCG-3'

Probe: 5'FAM - CAAATAACGCCGCCGAACGCCT-3'BHQ1

Forward: 5'-TTTTAGGAGAAGGCGAGTCG-3'

Reverse: 5'-CTCCTTAACGTAATACTCTTCGTCG-3'

Probe: 5'FAM-TCTCGCGCTCGCAAACGACC-3'

${ }^{a}$ Ensembl gene accession number. ${ }^{b}$ Human genome assembly GRCh38/hg38. 'The RASSF1A TaqMan hydrolysis probe sequence contains the SNP rs4688725. APC, adenomatous polyposis coli; CYP26A1, cytochrome P450 family 26 subfamily A member 1; HOX, homeobox; RASSF1, Ras association domain family member 1; TBX15, T-box 15; TGF $\beta 2$, transforming growth factor $\beta 2$; ALU, Arthrobacter luteus element.

GP4 lesions were determined to contain an equal tumor cell percentage within the selected regions of analysis.

DNA methylation analysis. DNA was extracted from $10 \mu \mathrm{m}$ sections of previously prepared FFPE blocks matching the selected H\&E slides using a QIAamp DNA Mini kit (Qiagen, Inc., Valencia, CA, USA), as previously described (23-25). DNA methylation was analyzed in the same GP4 area used for histological evaluation. Briefly, a total of 100-400 ng extracted DNA from the GP4 region for each GS7 case was bisulfite modified using EZ DNA Methylation Gold kit (Zymo Research Corp, Irvine, CA, USA), according to the manufacturer's protocol.

Methylated genes were detected using a MethyLight quantitative polymerase chain reaction (qPCR) assay that was conducted using the TaqMan ${ }^{\circledR}$ Gold with Buffer A pack (Thermo Fisher Scientific, Inc., Waltham, MA, USA), as described previously (23-25). The primer and probe sequences are presented in Table II. A percent methylation ratio (PMR) score was calculated to assess the methylation of each gene in GP4 separately for individual cases, as described previously, according to the formula of Eads et al (26): (Gene of Interest/ALU) sample/(Gene of Interest/ALU) CpGenome x100\%. CpGenome is a commercially available fully methylated DNA (EMD Millipore, Billerica, MA, USA). $A L U$ was a control reaction that measured the level of input DNA and normalized the signal for each methylation reaction (27).

Statistical analysis. The Mann-Whitney U test was performed to analyze the differences in the median continuous PMR across different clinicopathological categories. Pearson's Chi square test was used to analyze proportional differences in Cribriform and/or IDC status and clinicopathological 
Table III. Association between cribriform and/or IDC-positive $(n=61)$ and negative $(n=30)$ cases and clinicopathological features.

\begin{tabular}{|c|c|c|c|}
\hline \multirow[b]{2}{*}{ Parameter } & \multicolumn{2}{|c|}{ Cribriform and/or IDC } & \multirow[b]{2}{*}{ P-value } \\
\hline & Negative & Positive & \\
\hline \multicolumn{4}{|c|}{ Gleason score, $\%$ cases } \\
\hline $7(3+4)$ & 34.9 & 65.1 & \multirow[t]{2}{*}{$0.552^{\mathrm{a}}$} \\
\hline $7(4+3)$ & 28.6 & 71.4 & \\
\hline \multicolumn{4}{|c|}{ Pathological stage, $\%$ cases } \\
\hline $\mathrm{pT} 2$ & 46.7 & 53.3 & \multirow[t]{2}{*}{$0.007^{\mathrm{a}}$} \\
\hline pT3 & 20.0 & 80.0 & \\
\hline \multicolumn{4}{|c|}{ Surgical margins, $\%$ cases } \\
\hline Negative & 30.8 & 69.2 & \multirow[t]{2}{*}{$0.481^{\mathrm{a}}$} \\
\hline Positive & 38.5 & 61.5 & \\
\hline \multicolumn{4}{|l|}{ Age, $\%$ cases } \\
\hline Below median & 32.6 & 67.4 & \multirow[t]{2}{*}{$0.941^{\mathrm{a}}$} \\
\hline Above median & 33.3 & 66.7 & \\
\hline \multicolumn{4}{|c|}{ Preoperative PSA, $\%$ cases } \\
\hline Below median & 45.5 & 54.5 & \multirow[t]{2}{*}{$0.022^{\mathrm{a}}$} \\
\hline Above median & 22.0 & 78.0 & \\
\hline \multicolumn{4}{|c|}{ Prostate weight, \% cases } \\
\hline Below median & 38.1 & 61.9 & \multirow[t]{2}{*}{$0.542^{\mathrm{a}}$} \\
\hline Above median & 31.8 & 68.2 & \\
\hline \multicolumn{4}{|l|}{ Median PMR } \\
\hline$A P C$ & 31.7 & 47.3 & $0.045^{\mathrm{b}}$ \\
\hline HOXD3 & 25.8 & 27.1 & $0.888^{\mathrm{b}}$ \\
\hline HOXD8 & 25.7 & 27.7 & $0.278^{\mathrm{b}}$ \\
\hline CYP26A1 & 16.3 & 18.5 & $0.426^{\mathrm{b}}$ \\
\hline RASSF1 & 69.5 & 99.2 & $0.007^{\mathrm{b}}$ \\
\hline$T B X 15$ & 10.0 & 21.6 & $0.013^{\mathrm{b}}$ \\
\hline$T G F \beta 2$ & 0.0 & 0.0 & $0.287^{\mathrm{b}}$ \\
\hline
\end{tabular}

Cribriform architecture and/or IDC in association with clinicopathological features and median PMR values within the exact same GP4 area. P-values were obtained using the ${ }^{a}$ Pearson's $\chi^{2}$ test and ${ }^{\mathrm{b}} \mathrm{Mann}-$ Whitney $\mathrm{U}$ test. IDC, intraductal carcinoma; PMR, percent methylation ratio; PSA, prostate-specific antigen; GP, Gleason pattern; APC, adenomatous polyposis coli; CYP26A1, cytochrome P450 family 26 subfamily A member 1; HOX, homeobox; RASSF1, Ras association domain family member 1; TBX15, T-box 15; TGF $\beta 2$, transforming growth factor $\beta 2$.

categories. All statistical analyses were conducted using SPSS version 21 software (SPSS, IBM Software, Armonk, NY, USA). For all stated methods, a two-sided value of $\mathrm{P} \leq 0.05$ was considered to indicate a statistically significant difference.

\section{Results}

Clinicopathological features associated with GP4 cribriform architecture and/or IDC. The gland morphology of 91 GP4 tumor specimens obtained from RPs with GS7 pathology composed of comparable proportion $(\geq 80 \%)$ of tumor cells was examined in the current study. The presence of a cribriform growth pattern was identified in the GP4 areas of $61 / 91(67.0 \%)$ GS7 cases, whereas IDC was present in 21/91 (23.0\%) GS7 cases. All 21 RP tissue specimens with IDC were also positive for cribriform architecture. Notably, the proportion of cases positive for cribriform architecture and/or
IDC did not significantly differ between GS7 cases composed predominantly of GP4 (GP4+3; where the proportion of GP4 is $>50 \%$ of the total pattern seen) vs. predominantly GP3 (GP3+4); however cribriform and/or IDC positive status was significantly associated with a more advanced pathological stage (pT3 vs. pT2; $\mathrm{P}=0.007$; Table III) and higher preoperative prostate-specific antigen levels (PSA; $\mathrm{P}=0.022$; Table III).

DNA hypermethylation in areas with GP4 cribriform architecture and/or IDC. Gene specific DNA methylation differences were investigated between GP4 tumor areas positive for cribriform and/or IDC, and cases that did not harbor these features. For all genes investigated, PMR values were increased in GP4+3 vs. GP3+4, and in pT3 vs. pT2 cases (Table III). However, a significant association was only observed between CYP26A1 PMR and GS (GP4+3 vs. GP3+4; $\mathrm{P}=0.004$; Table IV), as well as between RASSF1 and TBX15, and pathological stage (pT3 vs. pT2; $\mathrm{P}=0.027$ and $\mathrm{P}=0.011$, 
Table IV. Median PMR values of methylation markers and Mann-Whitney U P-values stratified according to Gleason score and pathological stage.

Median PMR values

\begin{tabular}{llllllll}
\cline { 2 - 5 } Parameter & APC & HOXD3 & HOXD8 & CYP26A1 & RASSF1A & TBX15 & TGFb2 \\
\hline Gleason score & & & & & & & \\
$\quad 7(3+4)$ & 40.0 & 24.8 & 25.5 & 15.3 & 92.3 & 16.5 & 0.0 \\
$7(4+3)$ & 52.8 & 31.6 & 28.8 & 26.0 & 93.5 & 25.7 & 0.2 \\
$\quad$ Mann-Whitney U P-value & 0.120 & 0.084 & 0.492 & 0.004 & 0.523 & 0.220 & 0.779 \\
Pathological stage & & & & & & & \\
pT2 & 32.4 & 24.9 & 25.6 & 13.8 & 82.2 & 11.4 & 0.0 \\
pT3 & 46.6 & 27.3 & 26.9 & 18.8 & 100.1 & 27.8 & 0.0 \\
Mann-Whitney U P-value & 0.082 & 0.272 & 0.738 & 0.227 & 0.027 & 0.011 & 0.397 \\
\hline
\end{tabular}

PMR, percent methylation ratio; APC, adenomatous polyposis coli; CYP26A1, cytochrome P450 family 26 subfamily A member 1; HOX, homeobox; RASSF1, Ras association domain family member 1; TBX15, T-box 15; TGF $\beta 2$, transforming growth factor $\beta 2$.

respectively; Table IV). Among the seven genes investigated, GP4 with a cribriform/IDC component exhibited a significant increase in the median PMR for APC, RASSF 1 and TBX15 (Table IV). The median PMR of $A P C$ in cases negative for cribriform and/or IDC features was $31.7 \%$, whereas the median PMR was $47.3 \%$ in positive cases $(\mathrm{P}=0.045)$. The median PMR of RASSF1 was $69.5 \%$ in negative cases and $99.2 \%$ in positive cases $(\mathrm{P}=0.007) . T B X 15$ methylation also exhibited a significantly increased PMR in cribriform/IDC positive cases, as compared with in negative cases, with a median PMR of $10.0 \%$ in negative cases and $21.6 \%$ in positive cases $(\mathrm{P}=0.013)$. An increased median PMR for $A P C$ and $T B X 15$, but not for RASSF1, was also detected within GP3 areas of the same GS7 tumors that harbored cribriform/IDC features within GP4 areas $(\mathrm{P}=0.028, \mathrm{P}=0.025$ and $\mathrm{P}=0.280$, respectively). Due to a limited number of biochemical recurrence events in the cohort, the prognostic utility of these biomarkers could not be comprehensively evaluated in the current study.

\section{Discussion}

The effective management of patients with $\mathrm{PCa}$ is hindered by a lack of optimal, highly sensitive and specific biomarkers that are able to predict the risk of disease aggressiveness at the time of diagnosis (28). Therefore, PCa treatment decisions are made with limited, and at times conflicting, information (28). In the present study, prognostic DNA methylation biomarkers were incorporated along with histological evaluation of cribriform and IDC patterns, in order to examine their association. To the best of our knowledge, this is the first study to investigate DNA methylation aberrations in tumor areas consisting of specific tumor architectural features, including cribriform and/or IDC, which provide information on the aggressive potential of GP4 carcinoma glands in GS7 tumors (17). Aberrations to DNA methylation in certain GP morphologies require further investigation as it may improve personalized patient treatment by utilizing biomarker information. The DNA methylation of $A P C$,
CYP26A1, HOXD3, HOXD8, RASSF1, TBX15 and TGF 22 genes were investigated in the current study, as they have previously been revealed as promising prognostic biomarkers for $\mathrm{PCa}$ with the potential for clinical utility in independent patient cohorts (23-25).

Of the seven genes analyzed, the median methylation levels of $A P C, R A S S F 1$ and $T B X 15$ were significantly associated with cribriform architecture and/or IDC within the same GP4 regions of GS7 RP cases, harboring a similar proportion and purity of tumor cells. Increased methylation of $A P C$ and $T B X 15$, but not RASSF1, was also detected within GP3 areas of the same GS7 tumors, which harbored cribriform/IDC features within GP4 areas. Hypermethylation of APC, RASSF 1 and TBX15 in the presence of cribriform architecture and/or IDC suggests these molecular markers may serve as indicators of these architectural features. This provides novel evidence for a link between cribriform and/or IDC features and methylation biomarkers, and warrants further investigation of additional hyper- and hypo-methylation events in association with various architectural patterns in PCa. Notably, the presence of cribriform architecture and/or IDC, rather than the precise percentage of cribriform cells, was associated with increased $A P C, R A S S F 1$, and TBX15 methylation within the carcinoma area (data not presented). Given these results, it is proposed that there is an extensive increase in DNA methylation within cribriform/IDC architecture, as well as in the adjacent carcinoma tissue. This concept must be evaluated in future studies, as it may have clinically significant implications for the detection of DNA methylation-based biomarkers as surrogate indicators of cribriform and/or IDC patterns in biopsy tissue samples. This requires further investigation into the sensitivity and specificity of $A P C, R A S S F 1$ and TBX15 PMR, in order to discriminate between cribriform and/or IDC positive and negative GP4 cases. The detection of increased DNA methylation of APC, RASSF1 and TBX15, indicating high-grade disease, may facilitate personalized treatment strategies, including intense follow-up or a lower threshold for initiating adjuvant radiotherapy for patients. 
Future studies are also necessary to explore the potential for the identification of a cribriform/IDC-associated DNA methylation signature in easily accessible biofluids, such as urine samples, as it may serve as a surrogate to examine epigenetic events heralding cribriform/IDC architecture in the prostate. In particular, due to the likely potential of IDC cells to travel into the prostatic urethra via the antecedent prostatic ductal system, it may be valuable to investigate $A P C, R A S S F 1$ and $T B X 15$ hypermethylation in association with IDC features in post-digital rectal exam urine samples obtained from patients with PCa.

Notably, not all of the seven methylation biomarkers were associated with cribriform and/or IDC architecture, suggesting unique epigenetically regulated pathways may be involved in PCa progression and its associated morphology. However, the potential biological roles of APC, RASSF1 and TBX15 methylation in the formation of these clinicopathological entities are not yet well characterized, and must be investigated in further studies. It has been demonstrated that DNA methylation at the promoters of $A P C$ and $R A S S F 1$ tumor suppressor genes can decrease gene expression levels, thus inducing signaling pathways important in cancer biology, including MYC, ERK1/2 and p21; however, the role of TBX15 in cancer has not yet been characterized (29-34). Conversely, it is possible that DNA methylation events are not biologically associated with cribriform and/or IDC architecture, but are separate hallmarks of (epi)genetic derangement in PCa.

One limitation to the current study is that it was carried out using a restricted number of RPs. Another potential limitation of the study is that it is an association study between biomarkers and clinical indices, not PCa-associated mortality that may predict clinical benefit. This was an exploratory study aimed at acquiring novel insight into DNA methylation aberrations in specific PCa tumor architectures. Therefore, only RP tissue samples with an abundant quantity of carcinoma were used. The results of the current study warrant future analysis of larger multi-site retrospective cohorts in order to elucidate the clinical utility and predictive clinical benefit of DNA methylation and cribriform/IDC architecture in GP4 PCa, especially in a biopsy setting.

In summary, the association of seven DNA methylation markers with cribriform and IDC architecture was assessed in a series of 91 GP4 carcinoma glands in RP tissues with GS7 PCa. Presence and prevalence of cribriform and/or IDC architecture was confirmed in prostate tumors, similar to previous studies on architectural patterns in PCa RPs (10-17). Furthermore, a significant increase in $A P C, R A S S F 1$ and $T B X 15$ methylation in association with cribriform/IDC features was demonstrated. Future studies are required to further validate the clinical utility and functional relevance of DNA methylation events in cribriform and IDC architecture, and whether it should be routinely included in the RP pathology reports. Due to the challenges in the unequivocal identification of these clinicopathological entities by certain pathologists, the evaluation of these morphological features must be incorporated in the rapidly developing field of digitized histopathology $(18,19,34)$. This may provide clinically translatable imaging markers for PCa prognosis. External validation of these findings within a larger population with a longer follow-up time is, therefore, essential.

\section{Acknowledgements}

This study was supported by the National Cancer Institute of Canada/the Canadian Prostate Cancer Research Initiative (grant no. 18568); Movember Translation Acceleration Grant from Prostate Cancer Canada (grant no. 2014-01); the Ontario Graduate Scholarships; and the Campbell Family Fellowship award. The authors thank Mr Michael Nesbitt for organizing and maintaining the patient clinical information that was used in this study.

\section{References}

1. Jemal A, Bray F, Center MM, Ferlay J, Ward E and Forman D: Global cancer statistics. CA Cancer J Clin 61: 69-90, 2011.

2. Gleason DF: Classification of prostatic carcinomas. Cancer Chemother Rep 50: 125-128, 1966.

3. Albertsen PC, Hanley JA, Gleason DF and Barry MJ: Competing risk analysis of men aged 55 to 74 years at diagnosis managed conservatively for clinically localized prostate cancer. JAMA 280: 975-980, 1998.

4. Epstein JI, Allsbrook WC Jr, Amin MB and Egevad LL; ISUP Grading Committee: The 2005 international society of urological pathology (ISUP) consensus conference on gleason grading of prostatic carcinoma. Am J Surg Pathol 29: 1228-1242, 2005.

5. Epstein JI, Partin AW, Sauvageot J and Walsh PC: Prediction of progression following radical prostatectomy. A multivariate analysis of 721 men with long-term follow-up. Am J Surg Pathol 20: 286-292, 1996.

6. Siadat F, Sykes J, Zlotta AR, Aldaoud N, Egawa S, Pushkar D, Kuk C, Bristow RG, Montironi R and van der Kwast T: Not all Gleason pattern 4 prostate cancers are created equal: A study of latent prostatic carcinomas in a cystoprostatectomy and autopsy series. Prostate 75: 1277-1284, 2015.

7. Kovi J, Jackson MA and Heshmat MY: Ductal spread in prostatic carcinoma. Cancer 56: 1566-1573, 1985.

8. McNeal JE and Yemoto CE: Spread of adenocarcinoma within prostatic ducts and acini. Morphologic and clinical correlations. Am J Surg Pathol 20: 802-814, 1996.

9. Guo CC and Epstein JI: Intraductal carcinoma of the prostate on needle biopsy: Histologic features and clinical significance. Mod Pathol 19: 1528-1535, 2006.

10. Robinson BD and Epstein JI: Intraductal carcinoma of the prostate without invasive carcinoma on needle biopsy: Emphasis on radical prostatectomy findings. J Urol 184: 1328-1333, 2010.

11. Cohen RJ, Chan WC, Edgar SG, Robinson E, Dodd N, Hoscek S and Mundy IP: Prediction of pathological stage and clinical outcome in prostate cancer: An improved pre-operative model incorporating biopsy-determined intraductal carcinoma. Br J Urol 81: 413-418, 1998.

12. Wilcox G, Soh S, Chakraborty S, Scardino PT and Wheeler TM: Patterns of high-grade prostatic intraepithelial neoplasia associated with clinically aggressive prostate cancer. Hum Pathol 29: 1119-1123, 1998.

13. Van der Kwast T, Al Daoud N, Collette L, Sykes J, Thoms J, Milosevic M, Bristow RG, Van Tienhoven G, Warde P, Mirimanoff RO and Bolla M: Biopsy diagnosis of intraductal carcinoma is prognostic in intermediate and high risk prostate cancer patients treated by radiotherapy. Eur J Cancer 48: 1318-1325, 2012.

14. Dong F, Yang P, Wang C, Wu S, Xiao Y, McDougal WS, Young RH and Wu CL: Architectural heterogeneity and cribriform pattern predict adverse clinical outcome for Gleason grade 4 prostatic adenocarcinoma. Am J Surg Pathol 37: 1855-1861, 2013.

15. Kryvenko ON, Gupta NS, Virani N, Schultz D, Gomez J, Amin A, Lane Z and Epstein JI: Gleason score 7 adenocarcinoma of the prostate with lymph node metastases: Analysis of 184 radical prostatectomy specimens. Arch Pathol Lab Med 137: 610-617, 2013.

16. Kweldam CF, Wildhagen MF, Steyerberg EW, Bangma CH, van der Kwast $\mathrm{TH}$ and van Leenders GJ: Cribriform growth is highly predictive for postoperative metastasis and disease-specific death in Gleason score 7 prostate cancer. Mod Pathol 28: 457-464, 2015. 
17. Trudel D, Downes MR, Sykes J, Kron KJ, Trachtenberg J and van der Kwast TH: Prognostic impact of intraductal carcinoma and large cribriform carcinoma architecture after prostatectomy in a contemporary cohort. Eur J Cancer 50: 1610-1616, 2014.

18. Shah RB, Magi-Galluzzi C, Han B and Zhou M: Atypical cribriform lesions of the prostate: Relationship to prostatic carcinoma and implication for diagnosis in prostate biopsies. Am J Surg Pathol 34: 470-477, 2010

19. Iczkowski KA, Egevad L, Ma J, Harding-Jackson N, Algaba F, Billis A, Camparo P, Cheng L, Clouston D, Comperat EM, et al: Intraductal carcinoma of the prostate: Interobserver reproducibility survey of 39 urologic pathologists. Ann Diagn Pathol 18: 333-342, 2014.

20. You JS and Jones PA: Cancer genetics and epigenetics: Two sides of the same coin? Cancer Cell 22: 9-20, 2012.

21. Sharma S, Kelly TK and Jones PA: Epigenetics in cancer. Carcinogenesis 31: 27-36, 2010.

22. Strand SH, Orntoft TF and Sorensen KD: Prognostic DNA methylation markers for prostate cancer. Int J Mol Sci 15 : 16544-16576, 2014.

23. Kron KJ, Liu L, Pethe VV, Demetrashvili N, Nesbitt ME, Trachtenberg J, Ozcelik H, Fleshner NE, Briollais L, van der Kwast TH and Bapat B: DNA methylation of HOXD3 as a marker of prostate cancer progression. Lab Invest 90 : 1060-1067, 2010

24. Liu L, Kron KJ, Pethe VV, Demetrashvili N, Nesbitt ME, Trachtenberg J, Ozcelik H, Fleshner NE, Briollais L, van der Kwast TH and Bapat B: Association of tissue promoter methylation levels of APC, TGF 32 , HOXD3 and RASSF1 with prostate cancer progression. Int J Cancer 129: 2454-2462, 2011.

25. Kron K, Liu L, Trudel D, Pethe V, Trachtenberg J, Fleshner N, Bapat B and van der Kwast T: Correlation of ERG expression and DNA methylation biomarkers with adverse clinicopathologic features of prostate cancer. Clin Cancer Res 18: 2896-2904, 2012.
26. Eads CA, Danenberg KD, Kawakami K, Saltz LB, Blake C, Shibata D, Danenberg PV and Laird PW: MethyLight: A high-throughput assay to measure DNA methylation. Nucleic Acids Res 28: E32, 2000.

27. Campan M, Weisenberger DJ, Trinh B and Laird PW: MethyLight. Methods Mol Biol 507: 325-337, 2009.

28. Van der Kwast TH: Prognostic prostate tissue biomarkers of potential clinical use. Virchows Arch 464: 293-300, 2014.

29. He TC, Sparks AB, Rago C, Hermeking H, Zawel L, da Costa LT, Morin PJ, Vogelstein B and Kinzler KW: Identification of c-MYC as a target of the APC pathway. Science 281: 1509-1512, 1998.

30. Thaler S, Hähnel PS, Schad A, Dammann R and Schuler M: RASSF1 mediates p21Cip1/Waf1-dependent cell cycle arrest and senescence through modulation of the Raf-MEK-ERK pathway and inhibition of Akt. Cancer Res 69: 1748-1757, 2009.

31. Farin HF, Mansouri A, Petry M and Kispert A: T-box protein Tbx18 interacts with the paired box protein Pax 3 in the development of the paraxial mesoderm. J Biol Chem 283: 25372-25380, 2008.

32. Mayanil CS, George D, Freilich L, Miljan EJ, Mania-Farnell B, McLone DG and Bremer EG: Microarray analysis detects novel Pax3 downstream target genes. J Biol Chem 276: 49299-49309, 2001.

33. Esteller M, Sparks A, Toyota M, Sanchez-Cespedes M, Capella G, Peinado MA, Gonzalez S, Tarafa G, Sidransky D, Meltzer SJ, et al: Analysis of adenomatous polyposis coli promoter hypermethylation in human cancer. Cancer Res 60: 4366-4371, 2000.

34. Pfeifer GP and Dammann R: Methylation of the tumor suppressor gene RASSF1 in human tumors. Biochemistry (Mosc) 70: $576-583,2005$. 